\title{
Investigating the Effect of Drainage Tube Placement Depth on The Draining Effect of Chronic Subdural Hematoma Surgery
}

\author{
Xuejian Wang*, Xiangdong Li, Zhifeng Wang and Zhong Wang \\ Department of Neurosurgery, Affiliated Hospital 2 of Nantong University, Nantong University, Nantong, Jiangsu, PR China, 226001
}

*Corresponding author: Xuejian Wang, MD, Department of Neurosurgery, Affiliated Hospital 2 of Nantong University, Nantong University, Nantong, Jiangsu, PR China, 226001.
Received Date: July 02,2020

Published Date: July 15, 2020

\begin{abstract}
s
Background: To investigate the effect of drainage tube placement depth on the draining effect of drilling and drainage surgery on chronic subdural hematoma (CSDH).

Methods: 80 patients with CSDH who were admitted to our hospital from July 2017 to June 2019 were included in this study. They were divided into two groups, 40 cases in each group. In the observation group, the drainage tube was placed at $2 \mathrm{~cm}$ depth; in the control group, the drainage tube was placed to forehead direction, with a depth of $6 \mathrm{~cm}$. The drainage tube removal time ( $<2$ days, 2-5 days, $>5$ days), the incidence of intracranial gas accumulation, the incidence of complications, and the recurrence rate were compared between the two groups.

Results: The drainage tube removal time for observation group was: $<2$ days: 7 cases, 2-5 days: 24 cases, $>5$ days: 9 cases, and the drainage tube removal time for control group was: $<2$ days: 4 cases, 2-5 days: 28 cases, $>5$ days, 8 cases. The incidence of intracranial gas accumulation at 1 week after surgery was $15.0 \%(6 / 40)$ for observation group, which was higher than control group $(10 \%, 4 / 40)$. The incidence of postoperative complications was 5.0\% (2/40) for control group and 2.5\% (1/40) for observation group, but the differences were not statistically significant (P > $0.05)$. No recurrence was found in both groups at 1 month after surgery.

Conclusion: Based on this study, we found that enlarging the bone window exposure during CSDH surgery, repeated rinse of hematoma cavity until fluid is clear, and encouraging patients to move earlier after surgery have positive effects on the treatment outcome, but the placement depth of drainage tube has no significant impact on draining effect. It is recommended that the drainage tube should be placed within $2 \mathrm{~cm}$, in order to reduce the risk of accidental entry into brain tissue and damages on subdural hematomas.
\end{abstract}

Key words: Chronic subdural hematoma; Drainage tube; Depth; Effect

Abbreviations: CSDH: Chronic Subdural Hematoma; CT: Computerized Tomography

\section{Background}

Chronic subdural hematoma (CSDH) refers to the hematoma located between dura mater and arachnoid membrane for more than 3 weeks after trauma. It is one of the common diseases in neurosurgery, and it occurs more frequently in older people. Some patients have a history of trauma. The pathogenesis of CSDH is not completely understood. Drilling and drainage surgery is one of the effective methods for treating CSDH $[1,2]$. Although this surgical method is well known and has been widely used by neurosurgeons, there are still many surgical details that need to be investigated in specific operations, in order to achieve the best therapeutic effect. For example, whether to drill a single hole or double holes, whether to drill on forehead or top nodule, whether to flush the hematoma cavity and what temperature should be used, whether to place a single drainage tube or double drainage tubes and what depth should the drainage tube be placed, etc., these are all technical 
details that can affect the treatment outcome. So far, the effect of drainage tube placement depth on draining effect is still unclear. Here, we carried out a related study to address this issue.

\section{Methods}

\section{Ethics approval and consent to participate}

This research has been approved by the ethics committee of our department. Informed consent has been obtained and this investigation has been conducted according to the principles expressed in the Declaration of Helsinki. And the authors have obtained written informed consent of all the patients.

General information: The CSDH patients admitted to our department from July 2017 to June 2019 were divided into observation group and control group using the random number table method. For the observation group, the drainage tube placement depth was within $2 \mathrm{~cm}$; for the control group, the drainage tube was placed in forehead direction, with the depth of $6 \mathrm{~cm}$ or longer. There were 40 cases in observation group, 16 males and 24 females; their ages ranged from 52 to 77 years old, with an average age of $(64.23 \pm 6.26)$ years old; 34 cases had hematoma on one side and 6 cases were on both sides; the hematoma volume ranged from 60 to $120 \mathrm{ml}$, with an average of (63.27 \pm 11.24$) \mathrm{ml}$. For the 40 patients in control group, there were 19 males and 21 females, aged from 55 to 82 years old, with an average of $(65.32$ \pm 7.24 ) years old; 32 cases had hematoma on one side and 8 cases were on both sides; the hematoma volume ranged from 56 to 110 $\mathrm{ml}$, with an average of $62.34 \pm 10.74 \mathrm{ml}$. After examination, the general data of the two groups are comparable.

Treatment methods: All patients received routine CT scan before surgery to confirm the disease and record the characteristics of hematoma. If the hematoma signal was relatively uniform, then drilling and drainage surgery was performed. The top nodule was primarily chosen for localizing hematoma during surgery. If the top nodule was not involved, then the thickest layer was selected. The size of the bone window was $2 \sim 3 \mathrm{~cm}$. After the dura was electrocoagulated, it was cut open in the " + " cross manner. If the tension was high, the chronic subdural hematoma was slowly released. After the tension decreased, the dura mater and the hematoma capsule were completely cut open. The dura mater was suspended, and a 12 \# red urinary catheter was placed at the bottom of hematoma cavity. The other end of urinary tube was connected with a $50 \mathrm{ml}$ syringe needle to aspire the hematoma. After the hematoma volume was reduced, the syringe was filled with saline and slowly flushed the cavity; at the same time, the syringe direction was adjusted in order to rinse all directions. When the rinse liquid was almost clear, the silicone drainage tube was placed into the hematoma cavity toward the forehead direction. The depth of drainage tube was $2 \mathrm{~cm}$ in observation group, and $6 \mathrm{~cm}$ in control group, with the direction toward forehead. In both groups, the side holes of drainage tubes were cut off; and the tubes were led out along the tunnel under scalp, and connected with enclosed drainage bags. After the surgery, patient head was placed in low position to drain liquid continuously. If the color of the drainage fluid was clear and the drainage volume was fast, then the drainage speed was reduced. The patients were encouraged to move earlier after surgery. Routine rehydration was performed with 1500 to $2500 \mathrm{ml}$, and the time of drainage tube removal was determined based on the results of CT reexamination. The patients were followed up for 1-3 months after operation to check hematoma recurrence.

Evaluation index: Skull CT reexamination was performed at 24 hours, one week, and one month after the operation. Extubation time: the drainage tube could be removed if most of the drainage (greater than 90\%) and space occupying effect were eliminated, and the color of drainage fluid became lighter. Complication rate and recurrence rate were monitored.

Statistical analysis: SPSS 17.0 statistical software was used to process the data. Measurement data were expressed as mean \pm standard deviation ( $\mathrm{x}- \pm \mathrm{s}$ ) and analyzed with $\mathrm{t}$ test. The count data was analyzed using $\chi 2$ test. Fisher exact probability method was used for data with $\mathrm{n}<5$. $\mathrm{P}<0.05$ was considered statistically significant.

\section{Results}

There was no significant difference in draining effect between the two groups: most of the patients were fully drained within 2-5 days and the drainage tube was then removed. The drainage tube removal time of the two groups were: observation group: <2 days: 7 cases, 2-5 days: 24 cases, $>5$ days: 9 cases; control group: $<2$ days: 4 cases, 2-5 days: 28 cases, $>5$ days: 8 cases. There was no significant intracranial gas accumulation or tension pneumonia in both groups. The incidence of intracranial gas accumulation at one week after surgery was $15.0 \%$ (6/40) for the observation group, which was higher than the control group $(10 \%, 4 / 40)$. The gas accumulation was completely absorbed at 1 month after surgery, as examined by CT scan. There were 1 case $(2.5 \%)$ of complications occurred in the observation group and 2 cases (5\%,2/40) in control group, which was not significantly different $(\mathrm{P}>0.05$, Fisher exact probability method). One patient in the control group developed epidural hematoma after surgery. We gave conservative treatment to this patient, and after a month, his hematoma was absorbed. One patient in the observation group and one patient in the control group developed connections between subdural hematoma and subarachnoid space, and they both had large drainage volume of clear cerebrospinal fluid. For these patients, we controlled the draining speed and gave them conservative treatments. After that, the patient conditions were improved, and no hematoma recurred. At the 1-month follow-up, CT reexamination showed no recurrence in all the patients, so the recurrence rate was the same for both groups. 


\section{Discussion}

CDSH is a common and frequently-occurred disease in clinical neurosurgery, but the cause of this disease is still not fully understood $[1,3,4]$. Currently, drilling and drainage surgery is accepted as an effective surgical method for CDSH [5-8]. But there is still a lot of controversy regarding the operation details of this method. For example, there is no consensus on the timing of the drainage tube placement and the number of drainage tube placement, etc. [9-12]. In theory, placing the drainage tube in the forehead can reduce intracranial gas accumulation, because it is at the topmost position of the hematoma cavity, which is effective and preferential to drain gas accumulation. However, after the drainage tube is blindly placed, whether the tube tip is at the topmost position, and whether adherence affects drainage effect are not certain. It is also possible that when the drainage tube is not placed under direct vision, the tube may damage the drainage vessels and enter the brain parenchyma by mistake, which can cause bleeding Due to these uncertain factors, we designed the current study to investigate the effect of drainage tube placement depth on the draining effect of CDSH surgery.

We used random number table to group the patients, and compared the difference in treatment effect related to drainage tube placement depth. The drainage tubes of the two groups were both placed in the direction of forehead top in supine position. The patients were divided into two groups based on the drainage tube depth. In observation group, the tube was about $2 \mathrm{~cm}$ from the edge of bone window. In control group, the tube was placed in the direction of forehead with a depth of $6 \mathrm{~cm}$. The similarities and differences of draining effect between the two groups were recorded. In order to avoid blind placement of the tube, which might damage brain tissue, we opened a $2-3 \mathrm{~cm}$ bone window in these patients. The relatively big bone window can help to avoid the brain damage induced by intracerebral hematoma, which may occur during the rinse with red urinary catheter and during the placement of drainage tube in control group.

There was no significant difference in drainage effect between the two groups. Most of the patients were fully drained within 2-5 days, and the drainage tube was then removed. There was also no significant difference in the extubation time between the two groups, indicating that the two different depths of intubation can both achieve good draining effects, which might be due to the following reasons: accurate surgical positioning and adequate drainage of hematoma cavity; repeated and thorough rinse until the drainage fluid was completely clear; there were multiple drainage openings on the drainage tube, so the hematoma was eventually drained from the back bone window as the brain tissue expanded. Theoretically, deep drainage tube placement can help the tube get closer to forehead, which can facilitate the drainage of intracranial gas accumulation. However, there was no significant difference in the incidence of intracranial gas accumulation between the two groups. The main reason may be that the patients were encouraged to move in the early stage, so they were constantly changing positions, which promoted rapid draining of liquids and gases.

Epidural hematoma occurred to one patient in control group after surgery. Considering that this case might be related to the fast drainage rate during or after surgery, we slowly released the hematoma for patients during the operation and slowly reduced the hematoma cavity pressure. By controlling the drainage speed after surgery, no other patients showed corresponding symptoms. This patient also became better after we controlled the drainage speed and used conservative treatment. There were two patients, one in observation group and one in control group, showed connections between subdural hematoma and subarachnoid space, with large drainage volume of clear cerebrospinal fluid. Considering that these cases might be related to the arachnoid damage caused during rinse and blind intubation, we controlled the drainage volume of these patients and gave them conservative treatment. Their conditions were improved afterwards, and no hematoma recurred in these 2 cases. Since both bridge vein injury and subdural effusion are causes for CDSH recurrence $[13,14]$, damages on bridge vein and arachnoid under blind or non-direct vision, and deep drainage tube placement may increase the corresponding risk. The use of neuroendoscopy can solve the problem of visibility to a certain extent $[15,16]$.

In summary, for patients with uniform density and nonorganized $\mathrm{CSDH}$, drilling and drainage surgery is an effective treatment. During the operation, the hematoma cavity should be fully and repeatedly rinsed until drainage fluid is clear, and the patients should be encouraged to move earlier. The depth of drainage tube placement has no significant effect on the draining effect. To reduce the risk of puncturing arachnoid membrane and accidentally entering brain tissue, we recommend to place the drainage tube at the edge of bone window to achieve a good draining effect.

\section{Conclusion}

Based on this study, we found that enlarging the bone window exposure during CSDH surgery, repeated rinse of hematoma cavity until fluid is clear, and encouraging patients to move earlier after surgery have positive effects on the treatment outcome, but the placement depth of drainage tube has no significant impact on draining effect. It is recommended that the drainage tube should be placed within $2 \mathrm{~cm}$, in order to reduce the risk of accidental entry into brain tissue and damages on subdural hematomas.

\section{Declarations}

\section{Consent for publication}

The authors have obtained all appropriate patient consent forms. In the form, the patient(s) has/have given his/her/their consent for his/her/their images and other clinical information to be reported in the journal. The patients understand that their names and initials will not be published and outstanding efforts 
will be made to conceal their identity, but anonymity cannot be guaranteed.

\section{Availability of data and material}

All the data have been included in this paper, no other data need to be shared.

\section{Competing interests}

None.

\section{Funding}

This work was supported by a grant from: Traditional Chinese medicine science and technology project in jiangsu province(YB2015113); The science and technology program of Nantong City (JC2018101); The science and technology program of Nantong Health Committee (MA2019003); The science and technology program of Nantong City (HS12018002). The funding source(s) had no more involvement.

\section{Authors' contributions}

We thank Dr XJ W for allowing inclusion of their patient in this study; XJ W for preparation of the manuscript; XD L and ZF W for technical assistance; Z W for direction.

\section{Acknowledgements}

This paper would not have been possible without the consistent and valuable reference materials that $\mathrm{i}$ have recevied from my supervisor, whose insightful guidance and enthusiastic encouragement in the course of my shaping this thesis definitely gain my deepest gratitude.

\section{References}

1. Borger V, Vatter H, Oszvald Á, Marquardt G, Seifert V, et al. (2012) Chronic subdural haematoma in elderly patients: a retrospective analysis of 322 patients between the ages of 65-94 years. Acta Neurochir (Wien) 154(9): 1549-1554.

2. Kageyama H, Toyooka T, Tsuzuki N, Oka K (2013) Nonsurgical treatment of chronic subdural hematoma with tranexamic acid. J Neurosurg 119(2): 332-337.

3. Ko BS, Lee JK, Seo BR, Moon SJ, Kim JH, et al. (2008) Clinical analysis of risk factors related to recurrent chronic subdural hematoma. J Korean Neurosurg Soc 43(1): 11-15.
4. Lee KS (2016) Chronic Subdural Hematoma in the Aged, Trauma or Degeneration? J Korean Neurosurg Soc 59(1): 1-5.

5. Mekaj AY, Morina AA, Mekaj YH, Manxhuka-Kerliu S, Miftari EI, et al. (2015) Surgical treatment of 137 cases with chronic subdural hematoma at the university clinical center of Kosovo during the period 2008-2012. J Neurosci Rural Pract 6(2): 186-190.

6. Liu W, Bakker NA, Groen RJ (2014) Chronic subdural hematoma: a systematic review and meta-analysis of surgical procedures. J Neurosurg 121(3): 665-673.

7. Mori K, Maeda M (2001) Surgical treatment of chronic subdural hematoma in 500 consecutive cases: clinical characteristics, surgical outcome, complications, and recurrence rate. Neurol Med Chir (Tokyo) 41(8): 371-381.

8. Weigel R, Schmiedek P, Krauss JK (2003) Outcome of contemporary surgery for chronic subdural haematoma: evidence based review. J Neurol Neurosurg Psychiatry 74(7): 937-943.

9. Regan JM, Worley E, Shelburne C, Pullarkat R, Watson JC (2015) Burr hole washout versus craniotomy for chronic subdural hematoma: patient out come and cost analysis. PLoS One 10(1): e0115085.

10. Liu W, Bakker NA, Groen RJ (2014) Chronic subdural hematoma: a systematic review and meta-analysis of surgical procedures. J Neurosurg 121(3): 665-673.

11. Santarius T, Qureshi HU, Sivakumaran R, Kirkpatrick PJ, Kirollos RW et al. (2010) The role of external drains and peritoneal conduits in the treatment of recurrent chronic subdural hematoma. World Neurosurg 73(6): 747-750.

12. Sikahall-Meneses E, Salazar-Pérez N, Sandoval-Bonilla B (2008) Chronic subdural hematoma. Surgical management in 100 patients. Cir Cir 76(3): 199-203.

13. Tanweer O, Frisoli FA, Bravate C, Harrison G, Pacione D, et al. (2016) Tranexamic Acid for Treatment of Residual Subdural Hematoma After Bedside Twist-Drill Evacuation. World Neurosurg 91: 29-33.

14. Wakuta N, Abe H, Nonaka M, Morishita T, Higashi T, et al. (2018) Analysis of Endoscopic Findings in the Chronic Subdural Hematoma Cavity: Bleeding Factors inChronic Subdural Hematoma Natural History and as Predictors of Recurrence. World Neurosurg S1878-8750(18)32901-2.

15. Shiomi N, Hashimoto N, Takeuchi H, Yamanaka T, Nakagawa N, Mineura K (2002) Endoscopic findings in chronic subdural hematoma. No Shinkei Geka 30(7): 717-722.

16. Yokosuka K, Uno M, Matsumura K, Takai H, Hagino H, et al. (2015) Endoscopic hematoma evacuation for acute and subacute subdural hematoma in elderlypatients. J Neurosurg 123(4): 1065-1069. 